\title{
Finite-size effects on pattern selection in immiscible fluids subjected to horizontal vibrations in weightlessness
}

\author{
P. Salgado Sánchez, ${ }^{1,{ }^{*}}$ Y. A. Gaponenko, ${ }^{2}$ J. Porter, ${ }^{1}$ and V. Shevtsova ${ }^{2}$ \\ ${ }^{1}$ Escuela Técnica Superior de Ingeniería Aeronáutica y del Espacio, Universidad Politécnica de Madrid, \\ Plaza de Cardenal Cisneros 3, 28040 Madrid, Spain \\ ${ }^{2}$ Microgravity Research Centre, CP-165/62, Université Libre de Bruxelles (ULB), av. F. D. Roosevelt, 50, B-1050 Brussels, Belgium
}

(Received 6 February 2019; published 26 April 2019)

\begin{abstract}
A detailed numerical investigation of frozen wave pattern selection in finite rectangular containers under microgravity conditions is presented. The column growth cycle controlling mode transitions is described and the instability diagram showing selected wave number as function of the vibrational velocity is obtained. In contrast to the continuous monotonic dependence of pattern wave number on forcing predicted by linear inviscid theory in the limit of infinitely long containers, the pattern selection process in finite domains is characterized by solution branches that persist over discrete forcing intervals. We describe how properties of the selected frozen wave pattern, including the finite critical forcing value, depend on container length. In long containers, a transition from nearly symmetric to asymmetric columnar development is found at sufficiently high forcing values, with the loss of (approximate) reflection symmetry evident in the mean flow associated with the transient growth of the pattern. The effect of container height is considered separately, in the limit of both shallow and deep layers. Shallow layers suppress the development of long-wave perturbations leading to higher wave-number patterns whose growth may be associated with an asymmetric series of collisions between developing columns and container boundaries. In thick layers, lower pattern wave numbers are observed and, although the column growth is on average more regular, the final state is often asymmetric. Finally, we compare our results with the dependence on container aspect ratio $\Gamma=L / 2 H$ predicted by inviscid theory. For $\Gamma \geq 2$, finite-size effects are reasonably weak and the numerically obtained thresholds are similar to but slightly higher than the theoretical values, most likely due to viscous effects. For $\Gamma \lesssim 2$, finite-size effects come to the fore and onset is significantly delayed.
\end{abstract}

DOI: 10.1103/PhysRevE.99.042803

\section{INTRODUCTION}

The dynamic response of an interface separating distinct fluids in the presence of external forces is of considerable interest in fluid dynamics, with a range of applications in engineering, biology, and physics. In the simple case where the excitation can be approximated as monochromatic, a number of distinct instabilities may arise, depending on the frequency, amplitude, and direction of the forcing. On earth, where gravity acts to flatten interfaces larger than the capillary length scale, the forcing is usually taken either to be vertical (perpendicular to the initial surface) or horizontal (parallel to it).

The case of vertical excitation can be treated as a modulation of the effective gravity in a comoving reference frame, and the isotropy and homogeneity of this parametric forcing mechanism lead to extended patterns characterized by a finite threshold. The subharmonic Faraday waves [1] that appear on gravitationally stable interfaces are well known. An enormous range of interesting patterns can be observed in such systems [2-5], especially if the monochromatic assumption is relaxed and multifrequency forcing is used. In the case of gravitationally unstable configurations, on the other hand, vertical vibration can act to suppress the Rayleigh-Taylor instability [6].

The case of horizontal excitation is somewhat more complicated to treat theoretically due to the lack of isotropy and the stronger effect of container boundaries. Since the fluids, with different densities, are accelerated towards or away from the solid (lateral) boundaries containing the contact line, they will move up and down near this boundary due to the difference in pressure. Such motion immediately induces (harmonic) waves at the forcing frequency, which disrupt the initially flat interface [7]. The oscillatory pressure gradient can also excite subharmonic waves, known as cross-waves [8], through a parametric forcing mechanism analogous to that of Faraday waves, but lacking the spatial homogeneity of the vertical case $[9,10]$. Depending on the extent to which these localized cross-waves penetrate inwards from the lateral walls, their interaction can induce temporal modulations $[9,11]$ and a range of secondary bifurcations [12].

The counterflow associated with the mass transfer occurring at the lateral walls in horizontally forced layers provides the mechanism for an instability of Kelvin-Helmholtz type $[13,14]$. The resulting interfacial waves, which are stationary in a comoving frame, are commonly referred to as frozen waves [15]. Theoretical studies in the large-aspectratio limit $[13,16]$, ignoring lateral boundaries and taking the initial interface to be flat, have determined the frozen wave 
threshold and the critical wave number. The instability may be supercritical or subcritical, depending on the density ratio, and associated with finite or vanishing wave number, depending on the layer depth. The predictions of the inviscid theory were critically examined in the work of Talib et al. [17], among others $[18,19]$, where it was shown numerically and experimentally that the threshold is underestimated for fluids of similar viscosity, and overestimated for fluids of high viscosity contrast.

Frozen waves are of increased importance in weightless environments, where the predicted threshold vanishes in the large-aspect-ratio limit $[13,20]$ for long-wave perturbations. The periodic stripe patterns found in supercritical $\mathrm{CO}_{2}$ in the experiments of Beysens et al. [21] were the first observation of the frozen wave instability in weightlessness; these studies of near-critical fluids were continued on ground using magnetic compensation [22,23]. More recent work [24] on supercritical fluids in microgravity examined thermovibrational instabilities, including the influence of finite-size effects.

Experiments conducted by Gaponenko et al. [25] showed that the frozen wave interfacial instability also exists for the diffusive boundary of miscible liquids with different densities and viscosities. Under the microgravity conditions of a parabolic flight, Gaponenko et al. [25] and Shevtsova et al. [26] showed that the sawtooth wave structure that first develops on the diffusive interface, and which occurs on ground as well, evolves in a similar manner as in experiments using immiscible liquids. The amplitude of the pattern grows until it reaches the upper and lower walls and the resulting structure can be described as a series of vertical columns of alternating liquids. Increasing the forcing still further can excite Faraday waves on the diffusive columnar interfaces, and thus produce an interfacial pattern with two distinct length scales [26]. The pattern selection process in this system has been further analyzed by Gaponenko et al. [27], who revealed a transition from columnar to vortical structures as the width of the initial diffusive interface increases (i.e., when density and viscosity gradients across the interface decrease).

Frozen wave experiments have also been performed in microgravity using immiscible liquids, and the recent work of Salgado Sánchez et al. [28] demonstrates that the interaction of various interfacial phenomena occurs in them as well. In particular, the appearance of interfacial patterns with two length scales, where Faraday waves are excited on the sides of a columnar structure that develops subsequent to the frozen wave instability, was confirmed in an immiscible two-fluid system for the first time [see Fig. 4(c)]. The interpretation of the results was affected by the imperfect and variable gravity level of the parabolic flight, the finite contact angle and associated dynamics, and other experimental limitations. In particular, the constraints of the container size clearly affected the results.

Given the importance of finite-size effects for understanding frozen wave patterns and related interfacial instabilities in microgravity, we undertake a systematic numerical investigation of these patterns in immiscible liquids for various container sizes and a range of forcing amplitudes (and thus selected wave numbers). Simulations are performed using parameters similar to those used by Salgado Sánchez et al. [28]

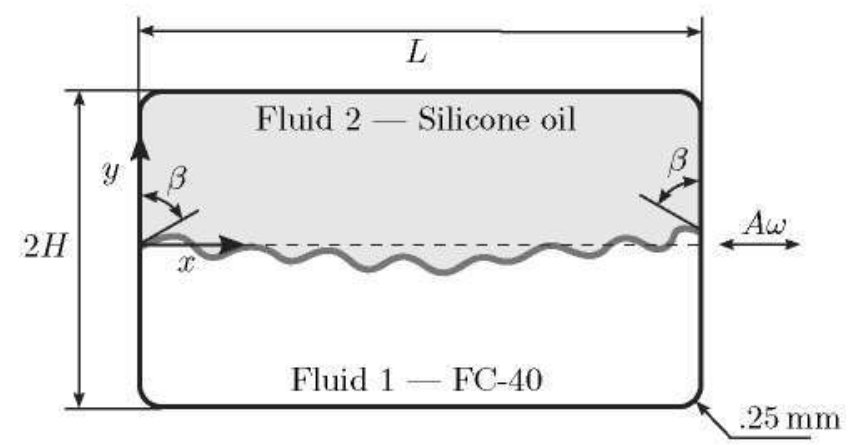

FIG. 1. Sketch illustrating the numerical model: two horizontal superposed layers of immiscible liquids confined in a $L \times 2 H$ rectangular container. The cell is subjected to vibrations of frequency $\omega$ and amplitude $A$ parallel to the initial flat interface (dashed line). Fluid behavior is analyzed for a pair of fluids similar to recent parabolic flight experiments [28].

to understand parabolic flight experiments, but differing, for convenience, in the viscosity ratio and contact angle. In contrast to the experiments of that paper, the frozen wave instability is analyzed here under ideal weightless conditions, with no residual gravity and with a perfectly flat initial interface oriented parallel to the vibrational axis.

The article is organized as follows. In Sec. II we discuss the mathematical formulation and numerical implementation of the problem. In Sec. III the development of the instability in weightlessness is detailed in finite containers, and the cycle of column growth is described and summarized in a stability diagram. In Secs. IV and V, the effects of length and height on different features of the instability, such as symmetry, are considered separately. In Sec. VI we compare our numerically obtained thresholds with the predictions of linear inviscid theory. Conclusions are given in Sec. VII.

\section{MATHEMATICAL FORMULATION}

The basic problem considered here is the application of horizontal vibrations to a rectangular container holding two superposed layers of immiscible liquids of equal volume and separated by an initially flat horizontal interface. The container has interior length $L$ and height $2 H$, as sketched in Fig. 1, and vibrates harmonically with amplitude $A$ and frequency $\omega$ in the $x$ direction.

We implement a level-set method [28-30] for tracking the movement of the interface, which is represented by the levelset variable $\phi$ obeying

$$
\phi_{t}+\nabla \cdot(\mathbf{u} \phi)=\gamma \nabla \cdot\left[\varepsilon \nabla \phi-\phi(1-\phi) \frac{\nabla \phi}{|\nabla \phi|}\right],
$$

where $\gamma=A \omega$ is selected as the vibrational velocity, and $\varepsilon$ is a small diffusive term added for numerical stability and taken to be on the order of the maximum mesh element size after convergence. This addition and that of the compression flux term $\phi(1-\phi) \nabla \phi /|\nabla \phi|$ are described in Olsson and Kreiss [29] and implemented by default in COMSOL Multiphysics.

Fluid properties are defined as functions of $\phi$ via the introduction of a (smoothed) Heaviside function $\mathcal{H}$ (as in 
Ref. [28]) by writing

$$
\begin{aligned}
& \rho=\rho_{1}+\left(\rho_{2}-\rho_{1}\right) \mathcal{H}, \\
& \mu=\mu_{1}+\left(\mu_{2}-\mu_{1}\right) \mathcal{H} .
\end{aligned}
$$

where

$$
\mathcal{H}(\phi)= \begin{cases}0,1 & \phi<-\varepsilon, \phi>\varepsilon \\ \frac{1}{2}+\frac{\phi}{2 \varepsilon}+\frac{1}{2 \pi} \sin \left(\frac{\pi \phi}{\varepsilon}\right) & |\phi| \leqslant \varepsilon .\end{cases}
$$

The subscripts 1 and 2 denote the two fluids and correspond to $\phi=0,1$, respectively, with the interface located at $\phi=0.5$.

We solve Eqs. (1) and (2) along with the continuous incompressible Navier-Stokes equations [31]

$$
\begin{aligned}
\rho\left[\mathbf{u}_{t}+(\mathbf{u} \cdot \nabla) \mathbf{u}\right] & =-\nabla p+\mu \Delta \mathbf{u}+\delta \mathbf{f}_{\sigma}+\rho \mathbf{G}(t) . \\
\nabla \cdot \mathbf{u} & =0 .
\end{aligned}
$$

The effect of interfacial tension at the immiscible interface is included in the momentum equation via $\mathbf{f}_{\sigma}$, defined by

$$
\mathbf{f}_{\sigma}=(\sigma \kappa) \mathbf{n}, \quad \kappa=\nabla \cdot \mathbf{n}, \quad \mathbf{n}=\frac{\nabla \phi}{|\nabla \phi|} .
$$

where $\kappa$ is the interface curvature, $\mathbf{n}$ is the unit normal vector and $\delta=|\nabla \phi|$ restricts the associated effects to the vicinity of the interface.

The applied acceleration is written as

$$
\mathbf{G}(t)=A \omega^{2} \cos (\omega t) \mathbf{i},
$$

where $\omega$ is the forcing frequency, $A \omega$ the vibrational velocity, and $\mathbf{i}$ the unit vector in the $x$ direction.

Navier-slip boundary conditions, applied on all solid walls, allow for fixing the contact angle and for motion of the contact line, which is observed in the relevant experiments [28]. This formulation, which includes an artificial frictional force $\mathbf{f}_{\nu}=\left(\mu / \delta_{\nu}\right) \mathbf{u}$ that scales with the viscous vibrational boundary layer $\delta_{\nu}$ is

$$
\mathbf{u} \cdot \mathbf{n}_{w}=0, \quad \mathbf{u}\left[\sigma\left(\mathbf{n}_{w}-\mathbf{n} \cos \beta\right) \delta\right]=0,
$$

where $\mathbf{n}_{w}$ is the corresponding normal vector.

At $t=0$ the liquids are assumed to be motionless $(\mathbf{u}=0)$ and separated by a flat interface. The vibrational forcing is initialized using the same type of smoothed Heaviside function, $\mathcal{H}$, with the transient time selected as $0.25 \mathrm{~s}$ for its relevance to the recent experiments of [28]. Similarly, fluid properties, which are listed in Table I, are similar to those

TABLE I. Physical properties of $20 \mathrm{cSt}$ silicone oil, FC-40, and the interface between them: density $\rho$, kinematic viscosity $\nu$, surface tension $\sigma$ (reproduced from Ref. [32]). and contact angle $\beta$ (selected to achieve an initial flat interface).

\begin{tabular}{lcc}
\hline \hline & $\rho\left(\mathrm{kg} / \mathrm{m}^{3}\right)$ & $\nu\left(\mathrm{m}^{2} / \mathrm{s}\right)$ \\
$20 \mathrm{cSt}$ silicone oil & 950 & $20 \mathrm{e}-6$ \\
$\mathrm{FC}-40$ & 1855 & $5 \mathrm{e}-6^{\mathrm{a}}$ \\
\hline & $\sigma(\mathrm{N} / \mathrm{m})$ & $\beta(\mathrm{rad})$ \\
FC-40 and silicone oil & $6.021 \mathrm{e}-3$ & $\pi / 2$ \\
\hline \hline
\end{tabular}

${ }^{\text {a The real kinematic viscosity is } 2.2 \mathrm{e}-6 \mathrm{~m}^{2} / \mathrm{s} \text {. It is increased to delay }}$ the appearance of subharmonic Faraday waves at high frequency. of the FC-40 and $20 \mathrm{cSt}$ silicone oil combination used in that reference. The values here differ from the actual experiment in that the viscosity of FC-40 is increased (by roughly a factor of 2 ) in order to delay the appearance of the secondary subharmonic surface wave mode [28], and the ideal contact angle of $\beta=\pi / 2$ is used to achieve a perfectly horizontal initial interface.

We use the following scalings for time, length, density, and viscosity,

$$
\left(\begin{array}{l}
t \\
\mathbf{x} \\
\rho \\
v
\end{array}\right) \rightarrow\left(\begin{array}{c}
\hat{t} \sqrt{\frac{\left(\rho_{1}-\rho_{2}\right) L^{3}}{\sigma}} \\
\widehat{\mathbf{x}} L \\
\widehat{\rho}\left(\rho_{1}-\rho_{2}\right) \\
\widehat{v} \sqrt{\frac{\sigma L}{\left(\rho_{1}-\rho_{2}\right)}}
\end{array}\right) .
$$

The dynamics contained in Eqs. (1)-(7) depend on the dimensionless vibrational velocity parameter $\mathcal{F}$ and frequency $\Omega$,

$$
\begin{gathered}
\mathcal{F}=A \omega \sqrt{\frac{\left(\rho_{1}-\rho_{2}\right) L}{\sigma}}, \\
\Omega=\omega L \sqrt{\frac{\left(\rho_{1}-\rho_{2}\right) L}{\sigma}},
\end{gathered}
$$

as well as the density and viscosity ratios, $\hat{\rho}$ and $\hat{v}$, the capillary number $\mathrm{Ca}$, and the aspect ratio $\Gamma$ :

$$
\widehat{\rho}=\frac{\rho_{2}}{\rho_{1}}, \quad \widehat{v}=\frac{\nu_{2}}{v_{1}}, \quad \mathrm{Ca}=v_{1} \sqrt{\frac{\left(\rho_{1}-\rho_{2}\right)}{\sigma L}}, \quad \Gamma=\frac{L}{2 H} .
$$

Recall that $H$ is the initial height of each fluid layer (see Fig. 1).

The commercial software COMSOL Multiphysics 5.3 is used for solving the two-dimensional governing equations (1)-(7) with the finite element method. Numerical convergence was previously tested for a similar configuration [28], and we make use of this to select the number of mesh elements and the interface thickness. Simulations throughout this paper are computed using a maximum element size $\mathcal{S}$ of

$$
\mathcal{S}=\min \left(\frac{2 H}{25}, \frac{7.5}{25}\right) \mathrm{mm}
$$

and

$$
\varepsilon=\frac{3}{2} \mathcal{S}
$$

A Generalized- $\alpha$ [33] integration scheme, in combination with both streamline [34] and crosswind [35] techniques is used. The maximum time step is set at $1 /(10 f)$, with step size reduced automatically when required for stability. Further details of the numerical scheme can be found in Ref. [28].

\section{DEVELOPMENT OF THE FROZEN WAVE INSTABILITY IN WEIGHTLESSNESS}

When two immiscible layers of fluid separated by a flat interface are subjected to horizontal vibrations, quasisteady 
undulations known as frozen waves appear at a critical forcing value. If lateral boundaries are ignored (i.e., the container is taken to be infinitely long) and the fluids are assumed to be deep and inviscid, then this threshold is given by [13]

$$
(A \omega)^{2}=\frac{\left(\rho_{1}+\rho_{2}\right)^{3}}{\rho_{1} \rho_{2}\left(\rho_{1}-\rho_{2}\right)} \sqrt{\frac{\sigma g}{\rho_{1}-\rho_{2}}},
$$

and the frozen wave has a wavelength $\lambda$ set by the capillary length scale:

$$
\lambda=2 \pi \sqrt{\frac{\sigma}{\left(\rho_{1}-\rho_{2}\right) g}} .
$$

In microgravity conditions, this critical wavelength becomes very large, and diverges in the limit of $g=0$ where the threshold also vanishes. Beyond lowering the threshold, the absence of the usual gravitational restoring force facilitates the growth of the initially sinusoidal patterns, which can rapidly develop into large columnar structures [22,23]. The length scale of these structures is determined by the fastest growing perturbation at the given forcing $[20,36]$ and the size constraints of the system (container).

We describe in Sec. III A how the frozen wave instability develops in finite containers subjected to vibrations in weightless environments, and how a cycle of column growth is key to the pattern selection process. The associated instability diagram is presented in Sec. III B.

\section{A. Column growth cycle and mode transitions}

In Fig. 2 two series of snapshots illustrate the time evolution of the instability in a container of size $L \times 2 H=$ $30 \mathrm{~mm} \times 7.5 \mathrm{~mm}$. The amplitude of the excitation is fixed at $A=1.5 \mathrm{~mm}$, while two different frequencies of (a) $f=45 \mathrm{~Hz}$ and (b) $60 \mathrm{~Hz}$ are used, corresponding to $\mathcal{F}=28.48$ and 37.97 , respectively. These figures focus on the evolution of the mean interface and velocity field (shown with scaled arrows) by averaging over one period of excitation. The harmonic surface waves, which are present near the lateral boundaries, are therefore filtered out and not visible.

Immediately after the forcing is initiated, the mean vertical velocity is especially large near the vertical walls, where the two liquids move up and down, and there is motion of the contact line as permitted by the Navier-slip boundary conditions [see Eqs. (7)]. The horizontal velocity on the interface is, on average, directed inwards $[37,38]$.

The net result of this initial agitation near the lateral walls is to provide a perturbation that activates column growth. These first columnar seeds can be seen near the lateral boundaries at $t=0.2 \mathrm{~s}$. As time passes, the frozen wave (column) growth moves inward toward the center of the container, as seen by the associated mean velocity; see the snapshots at $t=0.3,0.4 \mathrm{~s}$. The interfacial perturbations grow differently depending on their amplitude and the amount of liquid they involve. While the larger lateral columnar seeds grow until reaching the top and bottom walls, surface tension limits the growth of the smaller perturbation located in the center, which does not always reach the (upper and lower) container walls; see Fig. 2(a) at $t=0.9 \mathrm{~s}$. This can also be understood as a kind of wave-number frustration, where the more rapidly growing columns near the lateral walls, with wavelengths closer to the prediction of linear theory, induce a mismatch or defect in the center of the container.

The incomplete central column [Fig. 2(b) at $t=0.5,0.9 \mathrm{~s}$ ] is observed over a certain interval of the vibrational parameter. When it first appears, the associated fluid volume is small and it oscillates noticeably at the forcing frequency. This oscillatory motion, which is asymmetric, generates a mean flow that pushes it laterally (left or right) until it collides and merges with an adjacent column. In this scenario the central column is transient. As forcing increases, the wavelength of the lateral columns decreases and the central column grows in size. With more inertia, the harmonic oscillations diminish and the mean flow is more symmetric. In this scenario, the central column remains and can be located in the final structure.

As the forcing velocity is increased, the tip of the smaller column crosses the horizontal midline at $y=0$ (the location of the initial unperturbed interface) and we take this forcing value to characterize a mode transition. The wavelength of the pattern at this point, where the emerging column surpasses the horizontal midline, is also used to indicate the evolution of the pattern toward regularly spaced columns as this "local" wave number decreases by approximately a factor of two (see the snapshots at $t=0.9 \mathrm{~s}$ in Fig. 2).

The size of the incomplete column (defect) grows with increasing forcing. At a certain value it reaches the upper wall and, beyond that, its wavelength increases (i.e., the column width widens) with vibrational velocity. At some point its wavelength is too large and a new column seed appears, this time enclosing the other fluid and growing downward from the top. The repetition of this process defines the column growth cycle, which marks the transition from one mode to another and is driven by the increase in vibrational forcing and the associated selection of shorter wavelengths. This scenario characterizes the majority of results presented in this paper.

It is also notable for the forcing parameters shown in Fig. 2 that the column growth is approximately symmetric with respect to horizontal reflection about the middle of the container. This is evident in the mean velocity field, which is included in all except the last ( $t=0.9 \mathrm{~s}$ ) snapshots; when the selected steady state is reached the mean flow is null.

The final columnar structures are in qualitative agreement with the parabolic flight experiments of Salgado Sánchez et al. [28] in immiscible liquids [see Fig. 4(c)], with the experiments of Gandikota et al. [22,23] in supercritical fluid under magnetic compensation, and with the observations in microgravity of Gaponenko et al. [25], Shevtsova et al. [26], Gaponenko et al. [7] using miscible liquids; similar behavior is found in that case when the diffusive interface is thin and the density and viscosity gradients produce an effective interfacial tension [25].

\section{B. Pattern selection and instability diagram}

The wavelength selection process in weightlessness was considered by Lyubimova et al. [20] for the inviscid, infinitely extended problem, where the wavelength of the fastest growing perturbation was shown to increase continuously and monotonically with the vibrational parameter. When finite (longitudinal) size effects are relevant, however, numerical 
(a)
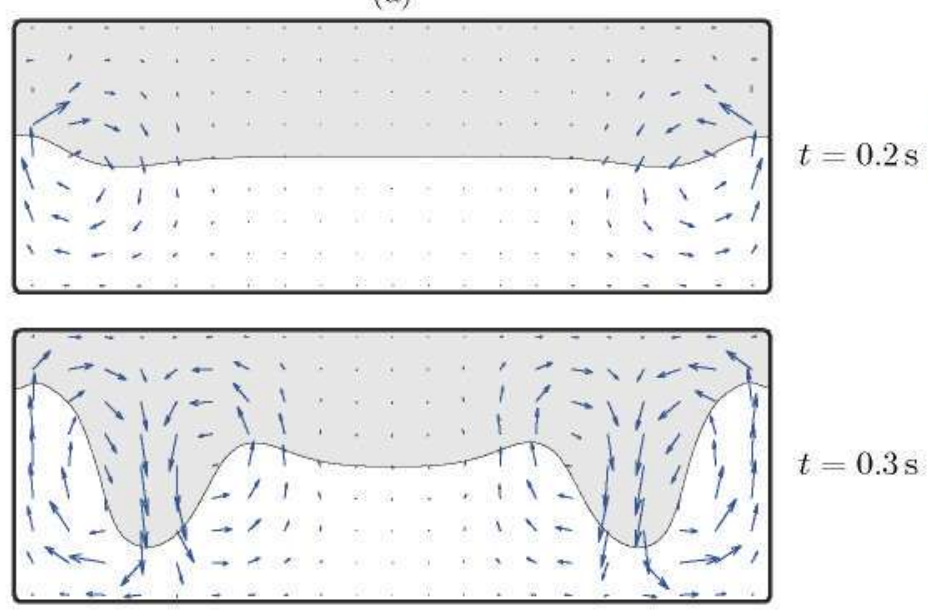

$t=0.3 \mathrm{~s}$
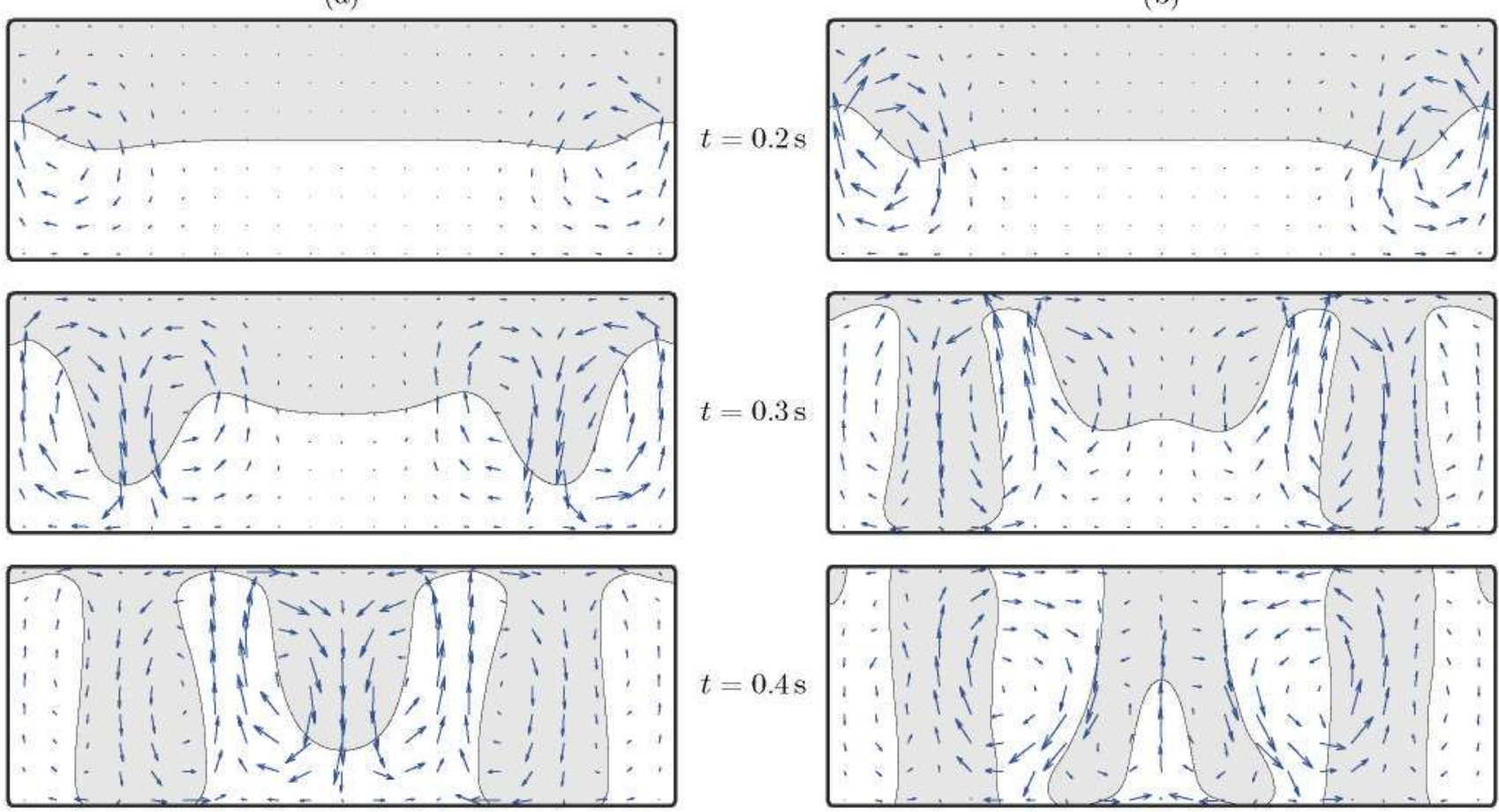

$t=0.4 \mathrm{~s}$
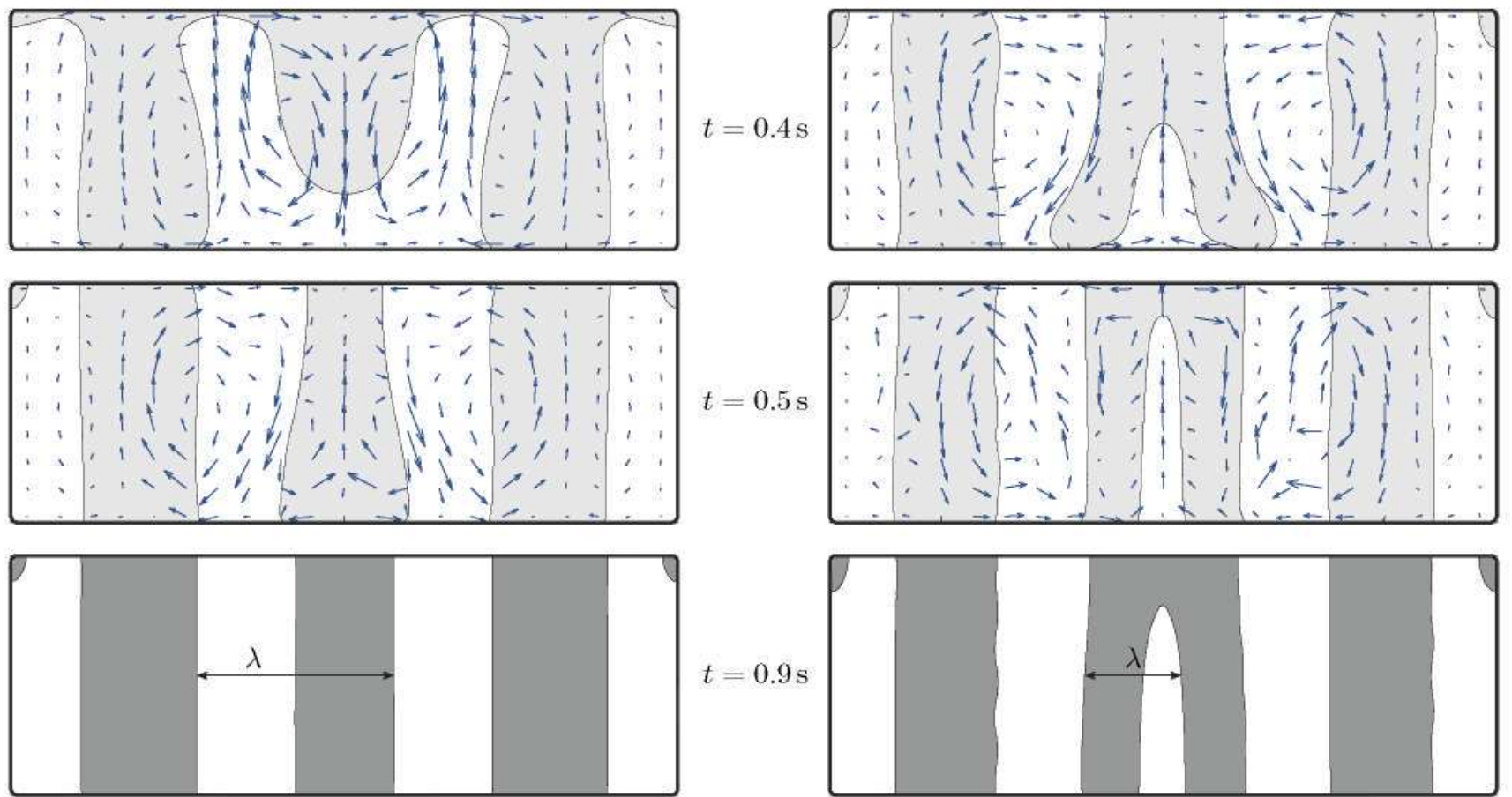

$f=45 \mathrm{~Hz}, A=1.5 \mathrm{~mm}$

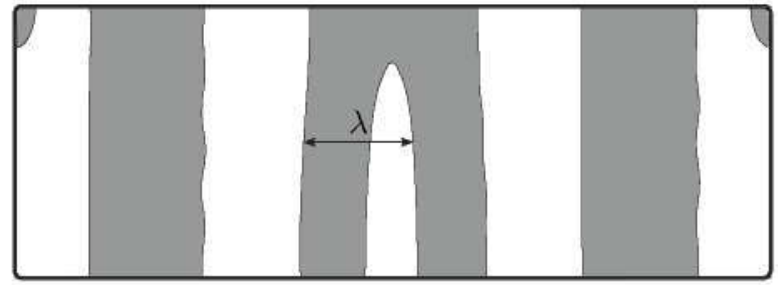

$f=60 \mathrm{~Hz}, A=1.5 \mathrm{~mm}$

FIG. 2. Time evolution, averaged over one excitation period, of the frozen wave instability under weightlessness in a container of size $L \times 2 H=30 \mathrm{~mm} \times 7.5 \mathrm{~mm}$ with $A=1.5 \mathrm{~mm}$ and two different frequencies: (a) $f=45 \mathrm{~Hz}$ and (b) $f=60 \mathrm{~Hz}$, corresponding to $\mathcal{F} \approx 28.48$ and $\mathcal{F} \approx 37.97$, respectively. The time is shown between the columns. The mean velocity field is shown using scaled arrows (scale not preserved between snapshots). The representative wavelength $\lambda$ in the defect region of each pattern is defined using the final snapshot at $t=0.9 \mathrm{~s}$. The appearance of subharmonic Faraday waves $[26,28]$ on the lateral boundaries of the columnar structure is also seen at that time in column (b).

simulations show that wave-number selection can differ significantly from these predictions.

To characterize the discontinuous selection process in finite length containers, we construct a mode instability diagram by measuring two relevant (dimensionless) wave numbers. One wavelength $\lambda$ is measured locally at the location (usually the center) of the emerging column (or defect) as illustrated in Fig. 2, with the dimensionless wave number defined as

$$
\mathcal{K}=\frac{L}{\lambda}
$$

The other is an average wave number $\langle\mathcal{K}\rangle$, equal to the number of paired columns (identifying lateral boundaries) in the columnar structure. This averaged wave number is used to label the mode of instability in the results that follow. Both quantities are calculated over a wide set of vibrational parameters $\mathcal{F}$ for a $30 \mathrm{~mm} \times 7.5 \mathrm{~mm}$ domain in Fig. 3. Snapshots are included showing selected final states for each mode of the instability.

At the onset of the frozen wave instability, the interface develops toward a single "pair" of columns, with the upper fluid (silicone oil) located in the center and the lower fluid (FC-40) divided nearly equally and aligned against the lateral 


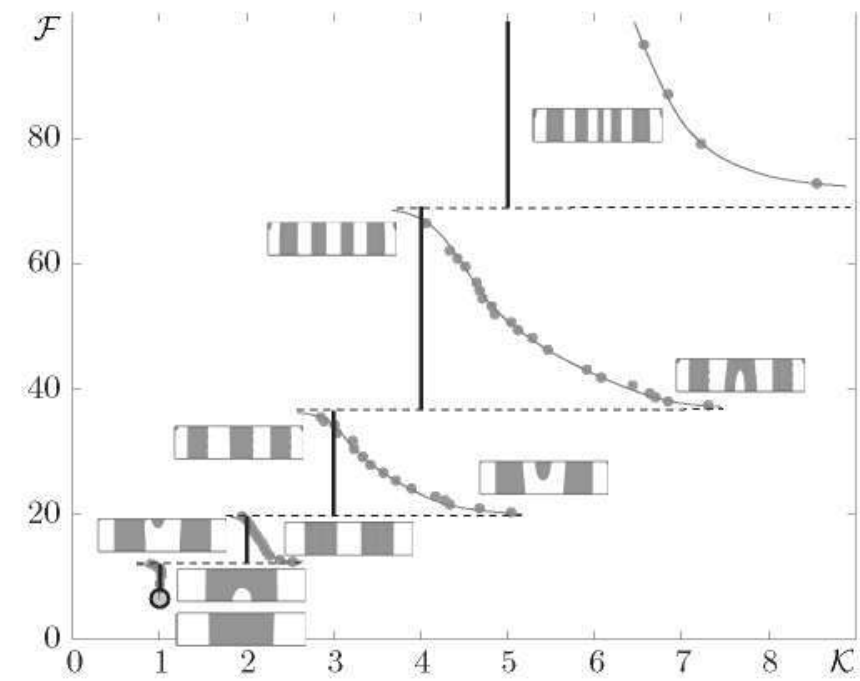

FIG. 3. Instability diagram in terms of the vibrational parameter $\mathcal{F}$ for a $L \times 2 H=30 \mathrm{~mm} \times 7.5 \mathrm{~mm}$ container showing the nondimensional averaged wave number $\langle\mathcal{K}\rangle$ (solid vertical lines), equal to the number of column pairs along the container length, and the wave number $\mathcal{K}$ (solid dots) near the defect in the central part of the container. The frozen wave threshold (see Sec. IV B) is distinguished by a circular gray marker. Insets illustrate the final pattern for selected points on each branch, while dashed lines provide a visual guide for the forcing interval associated with each mode.

walls; this is the $\langle\mathcal{K}\rangle=1$ mode. As the vibrational velocity is increased, the cycle of column growth described in Sec. III A is observed. Mode transitions are considered to occur when the small central column (defect) grows large enough to pass the horizontal midline $y=0$. As this happens, the surrounding column widens and splits apart.

For the range of vibrational velocities considered, there are five distinct modes, which are delimited by horizontal dashed lines in Fig. 3. Each mode is found over a certain interval of vibrational velocities, which widens as the wave number grows, indicating the increased cost of adding new columnar structures. In fact, in any real system, there is likely to be a point of wave-number saturation, or a transition to new phenomena, as the vibrational velocity becomes very large and acoustic or viscous effects assume a dominant role.

As mentioned above, the column growth cycle is often nearly symmetric with respect to reflection about the middle of the container. This approximate symmetry is observed over a range of low to medium strength excitations, especially in moderately sized containers where $\lambda$ remains on the order of the container length. Asymmetric behavior is also found, as will be discussed below, and the size of the container appears to play a crucial role in its emergence. We begin investigating the effect of container size on the frozen wave instability by varying the container length.

\section{EFFECT OF CONTAINER LENGTH}

Here we describe the dependence on container length of the instability diagram (Sec. IV A) and the frozen wave threshold (Sec. IV B). The appearance of symmetry breaking in larger containers is discussed (Sec. IV C) and, last, we suggest aspects of the transition from finite-sized containers to effectively infinite systems (Sec. IV D).

\section{A. Instability diagram with increasing length}

Figure 4 shows the frozen wave instability diagram in terms of (a) the nondimensional average wave number $\langle\mathcal{K}\rangle$ and (b) the local wave number $\mathcal{K}$ measured in the region of the emerging column. Results for containers lengths of $L=7.5 \mathrm{~mm}, 15 \mathrm{~mm}, 30 \mathrm{~mm}$, and $60 \mathrm{~mm}$, all with a fixed height $2 H=7.5 \mathrm{~mm}$, are displayed together, covering aspect ratios from $\Gamma=1$ to $\Gamma=8$. Container lengths are labeled in the figure for clarity.

The case of the shortest length $L=7.5 \mathrm{~mm}$ with $\Gamma=1$ differs from the scenario of column creation described previously (see Fig. 3 for comparison) since, over the range of forcing considered, the pattern selected is consistently the first mode $\langle\mathcal{K}\rangle=1$. There is just one branch of solutions over this range of the vibrational parameter because the development of more columns is prohibited by the (small) container size.

As expected, when the container length is increased, additional modes appear over the same range of the vibrational parameter. The interval over which each mode exists decreases with length, as does the absolute instability threshold itself, which is that of the largest permitted wavelength. In the largest container with $L=60 \mathrm{~mm}$ and $\Gamma=8$, numerous modes, up to $\langle\mathcal{K}\rangle=9$, can be excited. Furthermore, we observe a type of symmetry-breaking transition at $\mathcal{F} \sim 40$ where $\lambda / L \lesssim 0.2$; this is described in Sec. IV C.

To support the numerical results, Fig. 4(c) presents a snapshot from a parabolic flight experiment showing the frozen wave instability in microgravity in a $L \times 2 H=15 \mathrm{~mm} \times$ $7.5 \mathrm{~mm}$ container with $\mathrm{FC}-40$ and $20 \mathrm{cSt}$ silicone oil (see Table I). Horizontal vibrations are applied at $f=22 \mathrm{~Hz}$ with $A=1.755 \mathrm{~mm}$, corresponding to $\mathcal{F}=11.519$. The observed pattern is that of the $\langle\mathcal{K}\rangle=1$ mode, with a single pair of columns along the container. This is consistent with the simulations shown in Figs. 4(a) and 4(b) for $L=15 \mathrm{~mm}$ at that value of $\mathcal{F}$, despite the different contact angle and viscosity. Further comparison with available experiments is complicated by the irregularity of the microgravity level and the complex interaction between the frozen wave patterns, high-amplitude Faraday waves, and drop ejection [28].

\section{B. Effect of length on frozen wave threshold}

It was shown by Lyubimov and Cherepanov [13] and Lyubimova et al. [20] that in the absence of gravity, frozen waves arise at arbitrarily small vibrational forcing in deep and infinitely extended layers of inviscid fluids. Finite length domains, however, do not permit the associated long-wave perturbation and there is, in contrast, a finite threshold for the instability, even in the inviscid limit. This finite threshold results from, and is strongly affected by, the container length.

To get a more accurate estimate of the onset value, we measure the transient time $\tau$ for the interface amplitude at $x=L / 2$ to reach the bottom of the container. The depth $d$ of this growing column is shown as a function of time in Fig. 5(a) using a recipient of length $L=15 \mathrm{~mm}$ and height 

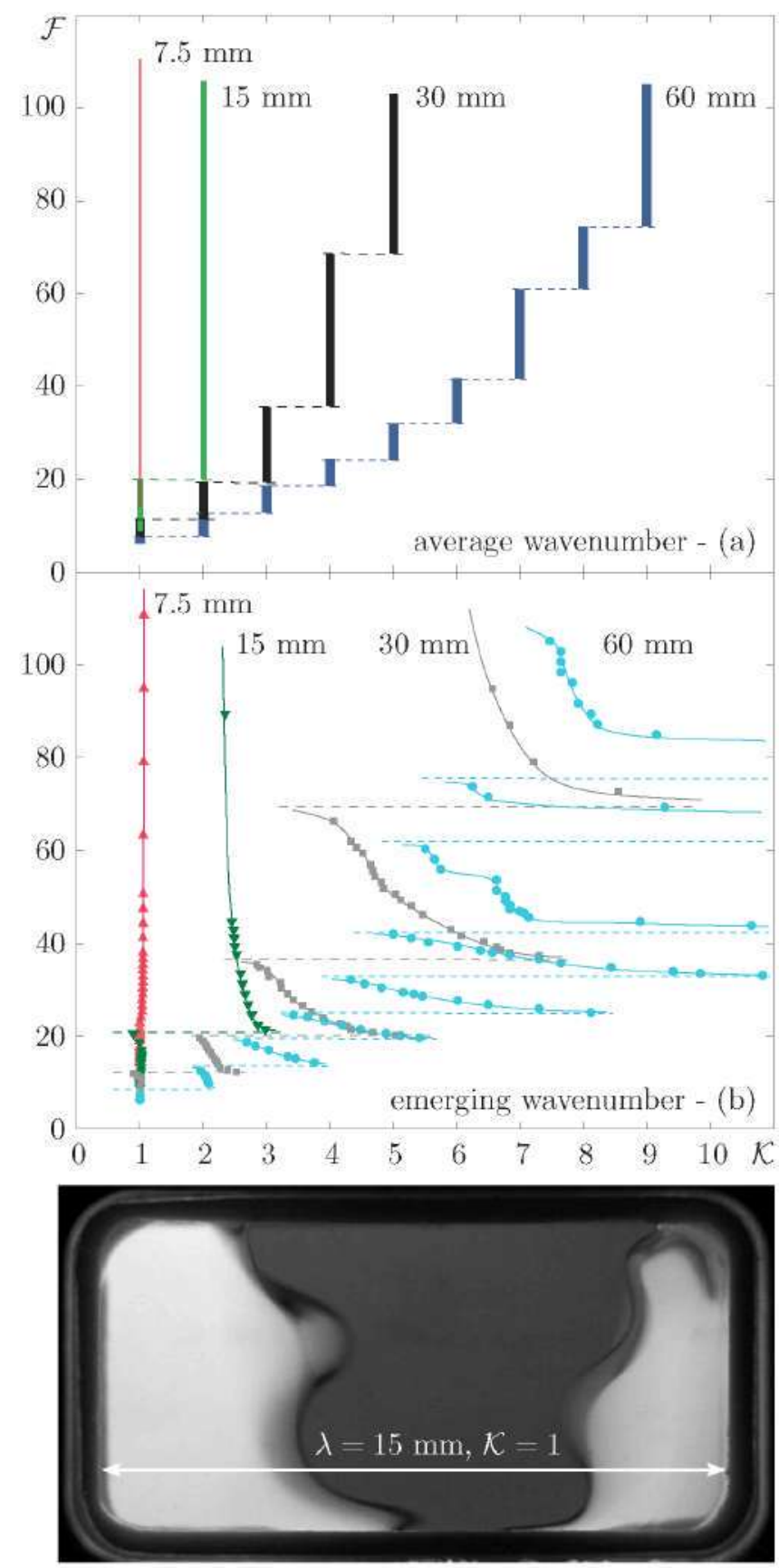

experimental snapshot - (c)

FIG. 4. (a, b) Instability diagrams in terms of the vibrational parameter $\mathcal{F}$ for fixed vertical dimension $2 H=7.5 \mathrm{~mm}$ and different container lengths (labeled in each curve): $L=7.5 \mathrm{~mm}$ (red, upright triangles), $15 \mathrm{~mm}$ (green, inverted triangles), $30 \mathrm{~mm}$ (black, squares), and $60 \mathrm{~mm}$ (blue, circles). (a) Average wave number $\langle\mathcal{K}\rangle$. (b) Wave number $\mathcal{K}$ near the defect region. Mode transitions are shown with dashed lines for visual reference. (c) Experiment showing a frozen wave pattern in microgravity in a $L \times 2 H=15 \mathrm{~mm} \times 7.5 \mathrm{~mm}$ container of FC-40 and $20 \mathrm{cSt}$ silicone oil subjected to horizontal vibrations at $f=22 \mathrm{~Hz}, A=1.755 \mathrm{~mm}, \mathcal{F}=11.519$.

$2 H=7.5 \mathrm{~mm}$, and for seven different forcing amplitudes just above onset. At least three different regimes can be clearly distinguished. First, there is an initial stage reflecting the
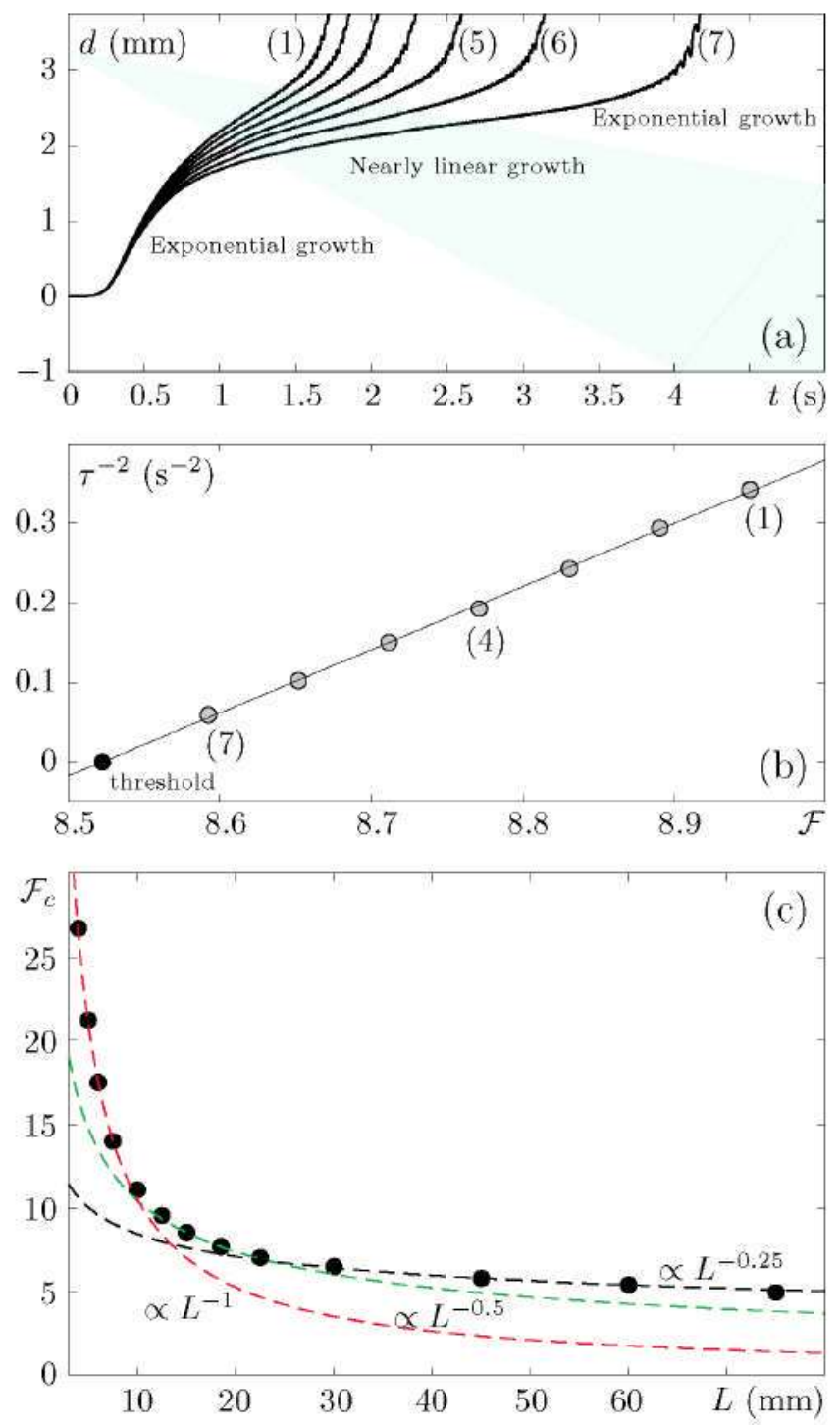

FIG. 5. (a) Time evolution of the interface depth $d$ (in $\mathrm{mm}$ ) at the center of a $L \times 2 H=15 \mathrm{~mm} \times 7.5 \mathrm{~mm}$ container for decreasing vibrational forcing (1-7) near threshold showing (at least) three distinct regions: Initial quasiexponential growth, a transition to nearly constant growth rate, and a transition to exponential growth when the column approaches the upper wall. (b) Inverse square of the frozen wave growth time, $\tau^{-2}$ (gray circles), for the smallest wave number $\langle\mathcal{K}\rangle=1$ as a function of the vibrational parameter $\mathcal{F}$. The linear dependence allows straightforward estimation of the instability threshold (black circle). Numbers refer to the different forcings used, as in panel (a). (c) Frozen wave instability threshold $\mathcal{F}_{c}$ as a function of the container length $L$ for fixed height $2 H=7.5 \mathrm{~mm}$. Fitting curves (dashed) assuming $L^{-1}, L^{-0.5}$, and $L^{-0.25}$ scalings are shown for comparison.

exponential growth of the linearized system as well as the timescale $(0.25 \mathrm{~s})$ of the smoothing function that modulates the forcing [see Eq. (3)]. After that, there is a transition to a nearly linear (constant growth) regime, which dominates the growth time of the column close to threshold. Finally, there is a more rapid (approximately exponential) acceleration toward the container ceiling. 


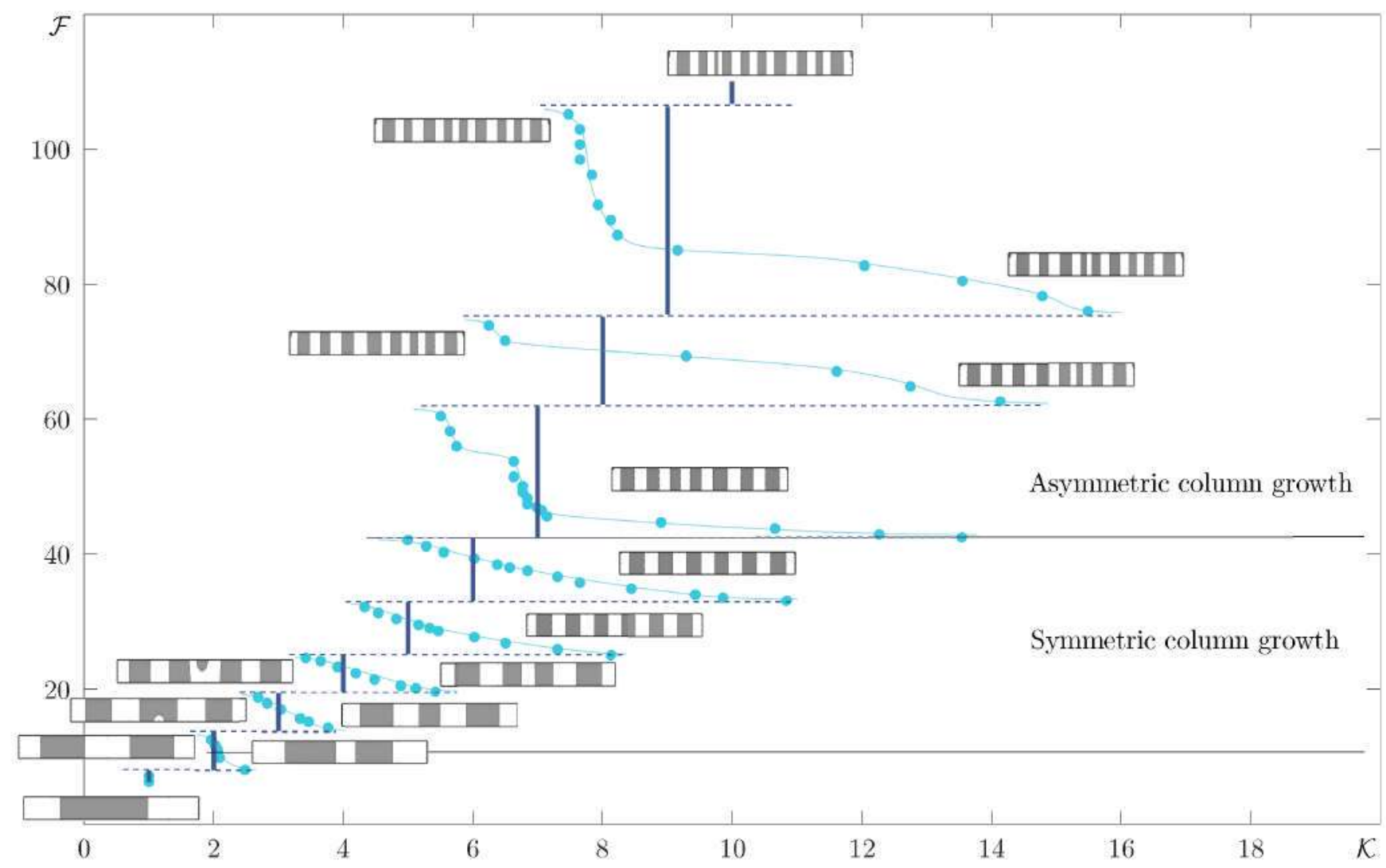

FIG. 6. Frozen wave instability diagram in terms of the vibrational parameter $\mathcal{F}$ for a $L \times 2 H=60 \mathrm{~mm} \times 7.5 \mathrm{~mm}$ container. The average wave number $\langle\mathcal{K}\rangle$ (vertical lines) gives the number of column pairs in the container, while the local wave number $\mathcal{K}$ (solid dots) near the defect region reflects the growth of this thin column (defect) into a regular column. Dashed lines are included for reference to delineate the mode transitions. A change from symmetric to asymmetric column growth (both regions are labeled) is observed near $\mathcal{F} \approx 40$.

We find that, despite the distinct growth stages involved, the inverse square of the total time needed for the column to reach the boundary $\tau^{-2}$ is proportional to $\mathcal{F}-\mathcal{F}_{c}$, as demonstrated in Fig. 5(b). In that figure, the data points (open circles) are the same as in Fig. 5(a) and the threshold (filled black circle) is estimated by a simple linear regression. Note that this procedure assumes there is no significant interval of bounded (small-amplitude) columns, which were never observed. The same method is used for different container lengths to construct Fig. 5(c), which also shows fitting curves (dashed) with scalings of $\mathcal{F}_{c} \propto L^{-1}, \mathcal{F}_{c} \propto L^{-0.5}$, and $\mathcal{F}_{c} \propto$ $L^{-0.25}$. These fits are shown mainly for the sake of comparison, but do suggest a slow scaling like $L^{-0.25}$ for the decrease of onset values as $L \rightarrow \infty$ where theory [13,20] predicts a vanishing threshold. In the limit of small containers, on the other hand, the frozen wave instability is delayed by the need for a large (dimensional) wave number and, thus, high vibrational velocity. The simulations in Fig. 5(c) suggest that $\mathcal{F}_{c}$ diverges as $L^{-1}$ in the limit $L \rightarrow 0$.

We note that threshold results for long large-aspect-ratio containers have a certain degree of uncertainty because, as discussed later in Sec. V A, the development of long wavelength modes $\langle\mathcal{K}\rangle=1,2, \ldots$ is affected by the upper and lower boundaries. Near onset, the calculation of the growth time for the first mode $\langle\mathcal{K}\rangle=1$ is more difficult, and sometimes not even possible. This problem, among other potential sources of numerical error, explains the lack of results for still larger $L$ values in Fig. 5(c) and may affect the threshold estimation for $L=75 \mathrm{~mm}$, which is slightly lower than the general trend would suggest.

\section{Symmetry breaking in the column growth cycle}

For sufficiently large containers, a transition from symmetric to asymmetric column growth is observed at moderate vibrational parameters. Figure 6 contains the instability diagram for $L=60 \mathrm{~mm}$ with insets showing selected final states along each branch.

Pattern selection at low vibrational velocities is characterized, in this domain, by cycles of nearly symmetric column growth, as described in Sec. III. Emerging columns develop in the middle of the container and remain there. When the vibrational parameter $\mathcal{F}$ reaches a value of about 40 , however, there is a transition to more asymmetric column growth. To illustrate this more clearly, we show the final steady density distribution along the horizontal midline in Fig. 7 as a function of the vibrational parameter.

As the pattern wavelength becomes smaller compared to the container length, lateral boundary effects become weaker; for $\mathcal{F} \approx 40$ we find $\mathcal{K}^{-1}=\lambda / L \lesssim 0.2$. This can also be interpreted as a weakening of the bias associated with the initiation of the forcing, which tends to perturb the interface 


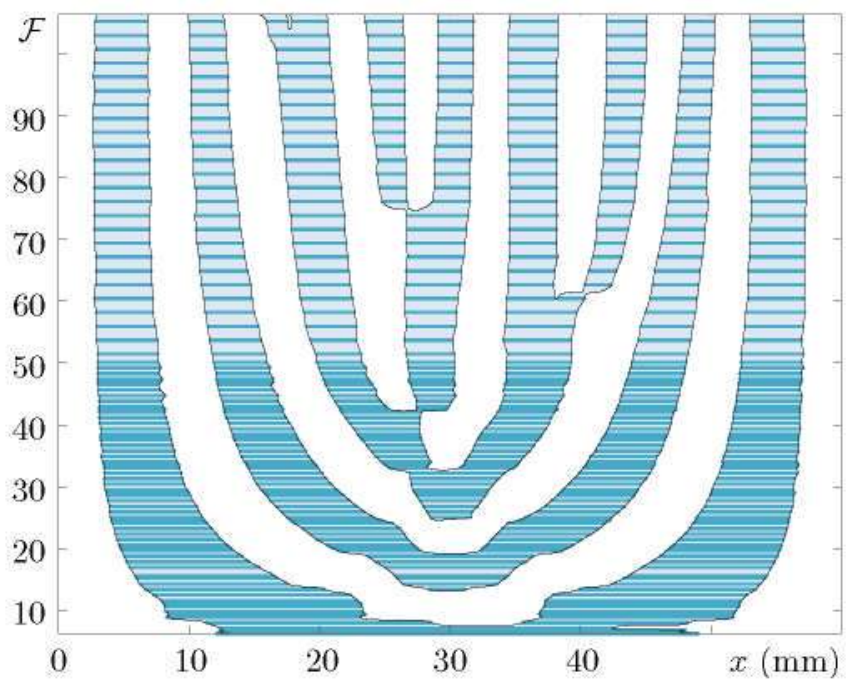

FIG. 7. Density distribution (silicone oil shaded) along the horizontal midline of the final steady state as a function of the vibrational parameter $\mathcal{F}$ for a rectangular container with $L \times 2 H=60 \mathrm{~mm} \times$ $7.5 \mathrm{~mm}$. Darker lines show the resolution used, which is higher for $\mathcal{F} \lesssim 50$. The column formation cycle becomes asymmetric at $\mathcal{F} \sim 40$.

more strongly near the lateral walls, causing the columns to grow more rapidly there. As the container length increases, the initial growth becomes more uniform across the interior and wave-number frustration can occur at various locations, not always at the center.

The mechanism of column creation in the asymmetric regime is more variable and complicated than in the symmetric case and often involves a type of fingering or branching during the transient stage as nascent columns of different size merge, either partly or completely. These fingers may branch off to the side of the main column at an angle only to be reabsorbed eventually, sometimes leaving drops behind, or they can reach the upper or lower boundary to form a new column. This process leads to the sudden appearance, in the final state, of a (pair of) new finite-width columns.

The location of the defect also varies, with a tendency to alternate from one side to the other, as seen in Fig. 7. The first asymmetric defect appears close to the center of the container. These defect locations, although seemingly random, are remarkably repeatable; indeed, so is the final columnar pattern itself, despite the complicated nonlinear dynamics that precedes it. Figure 7, which shows mainly continuous motion of the column boundaries versus $\mathcal{F}$, is constructed from a series of simulations that all start from the same initial condition of motionless fluids and a flat interface; the darker horizontal lines in this figure show the actual $\mathcal{F}$ values used, with the boundaries obtained by interpolation. The consistent defect locations across successive simulations are likely determined, at least in part, by the form of the forcing function.

Note that the measurement of the local (defect) wave number in the asymmetric regime must adjust to the changing location. We try to maintain continuity by switching from the region of one emerging column to the next when the measured column widths at the horizontal midline are comparable, i.e., after the first column has widened and developed, and before the next one appears.

Figure 7 makes clear that there is generally a distribution of column widths in the final state. Although this is not surprising given the complicated nonlinear dynamics involved in column growth and their collisions with the container walls (and with each other), the local wave-number variation seems to possess a degree of regularity as well. It can be seen in Figs. 6 and 7 that, while the energy barrier for adding new columns tends to increase with $\langle\mathcal{K}\rangle$, there is an apparent modulation of this trend when the column growth cycle switches from the symmetric (central) mechanism to the asymmetric one. That is, while the $\langle\mathcal{K}\rangle=7$ mode exists over a relatively wide range $(42.5 \lesssim \mathcal{F} \lesssim 61.5)$, the next mode, $\langle\mathcal{K}\rangle=8$, is found over a noticeably smaller interval $(61.5 \lesssim \mathcal{F} \lesssim 75)$. Following that, the $\langle\mathcal{K}\rangle=9$ mode is again observed over a large interval (75 $\lesssim \mathcal{F} \lesssim 106)$. This alternation, although inferred from limited data, suggests hysteresis in the asymmetric growth mechanism, and that it may be useful to consider asymmetric mode transitions in pairs; a more systematic investigation of this will be undertaken elsewhere.

When considering this symmetry-breaking transition, it is worth clarifying again that the symmetry referred to for the column growth cycle is an average, or approximate, one. The horizontal forcing is not symmetric on the fast timescale since the motion of the two lateral walls is out of phase by $\pi$, as are the harmonic waves generated by them. There is always a certain degree of asymmetry present in the columnar state, and this is amplified for the lowest $\langle\mathcal{K}\rangle=1$ mode near onset, as seen in Fig. 7 for $7 \lesssim \mathcal{F} \lesssim 10$. One reason for the greater asymmetry in the lowest mode is its interaction with the upper and lower walls, something that will be discussed more in Sec. VA.

\section{Approaching the infinitely extended case}

Considering the results described above for the frozen wave instability in microgravity, we can summarize the qualitative features suggested for the transition from finite to infinitely extended containers.

(1) The forcing interval associated with each frozen wave mode decreases, approaching zero (Sec. IV A);

(2) The absolute instability threshold decreases, approaching zero (Sec. IV B);

(3) The cycle of column growth that characterizes mode transitions becomes increasingly asymmetric, with defects appearing at various points along the container (Sec. IV C).

The first two of these statements are natural consequences of approaching the extended limit where arbitrary wave numbers are permitted and the threshold of long-wave perturbations is known to vanish for inviscid fluids [13]. The third is more speculative, since the appearance (or not) of defects in the infinite system, where discrete mode transitions do not generally occur, is unknown. It may be that the asymmetric column growth cycle observed here at larger forcing values eventually fades, giving way to more regular and uniform patterns. 


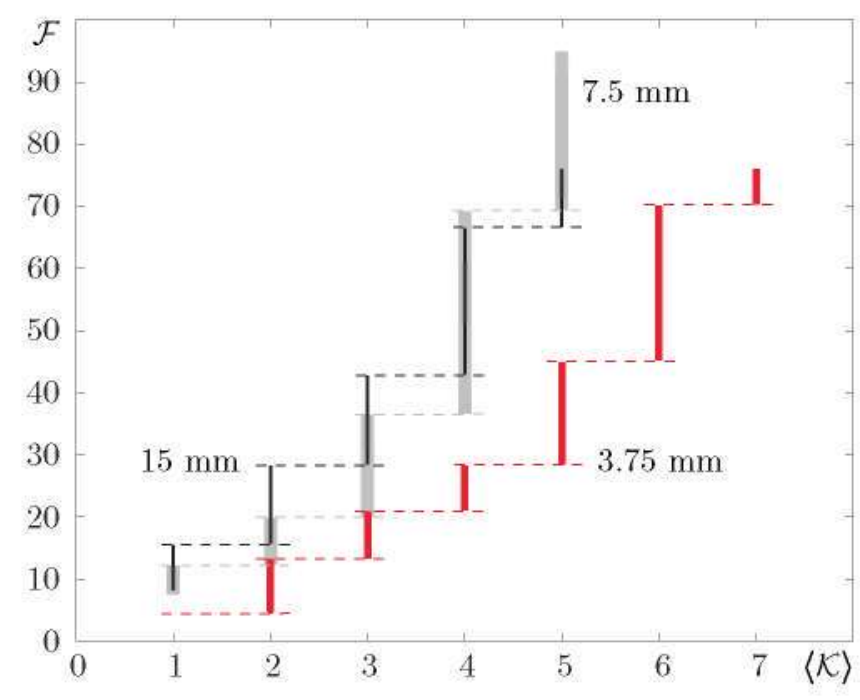

FIG. 8. Nondimensional wave number $\langle\mathcal{K}\rangle$ of the selected columnar pattern versus the vibrational parameter $\mathcal{F}$ for $L=30 \mathrm{~mm}$ and different container heights (labeled): $2 H=3.75 \mathrm{~mm}$ (red, medium thickness), $7.5 \mathrm{~mm}$ (gray, thicker), and $15 \mathrm{~mm}$ (black, thinner), Container heights are labeled for reference.

It is also worth noting that the scaling of $\mathcal{F}$ with $\mathcal{K}$ observed here, which is very roughly $\mathcal{F} \propto \mathcal{K}^{2}$ (see, e.g., Fig. 6) is different, at least over the range of parameters considered, than that predicted by Lyubimova et al. [20] for extended domains and inviscid fluids: $\mathcal{F} \propto \mathcal{K}$ for small $k$ and $\mathcal{F} \propto \sqrt{\mathcal{K}}$ for large $k$. This suggests (see Fig. 8 of Ref. [20]) that, besides finite-size effects, viscosity may be playing a crucial role.

\section{EFFECT OF CONTAINER HEIGHT}

In addition to the effects that finite length has on wave-number selection, mode transitions, and defect growth, the development of the frozen wave instability is also significantly affected by the container height. We describe here how the instability diagram changes if the length $L$ is fixed and the height $2 H$ is varied. The development of small wave-number patterns in thin containers is frustrated by collisions with the boundaries, as discussed in Sec. VA, while thick containers promote more asymmetry in the final frozen wave state, as discussed in Sec. VB.

Figure 8 shows the instability diagram in terms of the average wave number $\langle\mathcal{K}\rangle$ for fixed length $L=30 \mathrm{~mm}$, and different container heights: $2 H=3.75 \mathrm{~mm}, 7.5 \mathrm{~mm}$, and $15 \mathrm{~mm}$. As the container height is increased, the interval of the vibrational parameter $\mathcal{F}$ over which each mode is observed tends to increase as well (the addition of a new column requires more energy). The pattern selection process is generally similar to that described in Sec. IV with increasing $\mathcal{F}$, although there are differences when the height is much less, or much greater, than the wavelength. For $2 H=3.75 \mathrm{~mm}$, for instance, the thin container frustrates the development of the largest wavelength pattern, $\langle\mathcal{K}\rangle=1$, which is found only over a very small range of $\mathcal{F}$. In this domain, the second mode, $\langle\mathcal{K}\rangle=2$, exists over a much wider range; an explanation for this is given below.

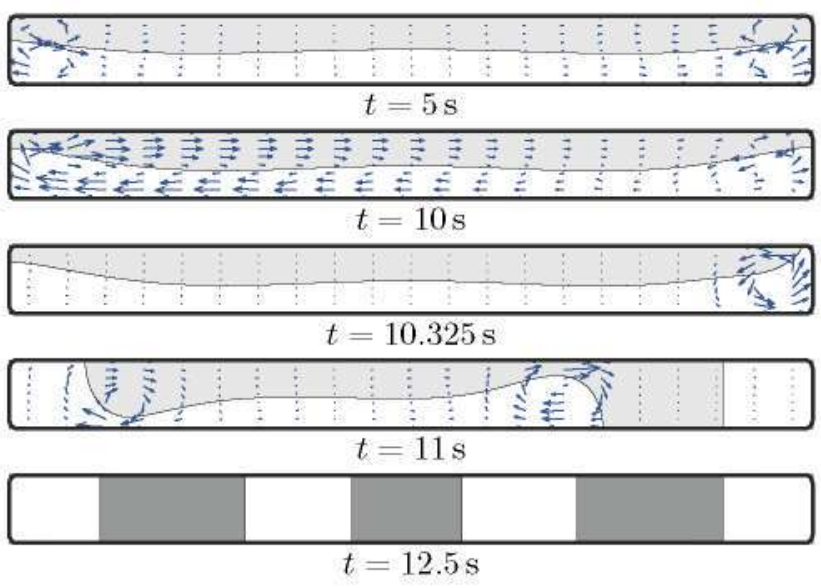

FIG. 9. Evolution of the frozen wave instability (averaged over one excitation period) in weightlessness in a $L \times 2 H=30 \mathrm{~mm} \times$ $1.875 \mathrm{~mm}$ rectangular container vibrated with $f=12 \mathrm{~Hz}$ and $A=$ $1.5 \mathrm{~mm}$, corresponding to $\mathcal{F} \approx 7.59$. Snapshots are labeled by time after initiation. The mean velocity field is shown in all but the final snapshot (scaling of arrow length is not constant between snapshots). Note the markedly asymmetric evolution of the interface, especially after the first (right) corner of the cell is covered by FC-40.

\section{A. Frozen wave instability in shallow containers: Suppression of small wave numbers}

When the height of the container is sufficiently small, the patterns of lowest wave number, $\langle\mathcal{K}\rangle=1,2$, etc., are frustrated before they can fully develop. Low frequency vibrations, particularly if the forcing amplitude $A \sim H$, enhance the harmonic motion produced by the endwalls. This harmonic excitation, as mentioned, is out of phase at the left and right walls. As soon as the amplitude near one (or both) of the endwalls is large enough for the interface to contact the upper wall, the dynamics is controlled in large part by surface and contact forces, and a column rapidly forms along the lateral wall. This process repeats, usually in alternating (asymmetric) fashion, inward along the container and, taken together, acts to select a pattern of higher average wave number than the initial perturbation.

Figure 9 illustrates this process in a $L \times 2 H=30 \mathrm{~mm} \times$ $1.875 \mathrm{~mm}$ container vibrated at $12 \mathrm{~Hz}$ with $A=1.5 \mathrm{~mm}$ (note $A>H$ ). When the excitation has been applied for $5 \mathrm{~s}$, a long wavelength perturbation is clearly visible on the interface. It grows in amplitude, especially near the endwalls, something that is likely promoted by the vibroequilibria effect, wherein the inhomogeneous oscillatory velocity field drives a reorientation of the interface that, for a horizontally vibrated interface, causes the heavier fluid to move up along the sides, leaving a trough in the center [39-41]. Harmonic waves (at the forcing frequency) are also excited, though these cannot be seen in the figure due to the averaging over one period. Eventually, one contact line traverses the upper corner of the cell and the interface dynamics is then locally driven by surface and contact forces acting to maintain the prescribed contact angle with the upper boundary. In this region undergoing rapid motion of the contact line, the average velocity field is considerably larger than in the rest of the container, as seen at $t=10.325 \mathrm{~s}$. Shortly after, the opposite (left) side 


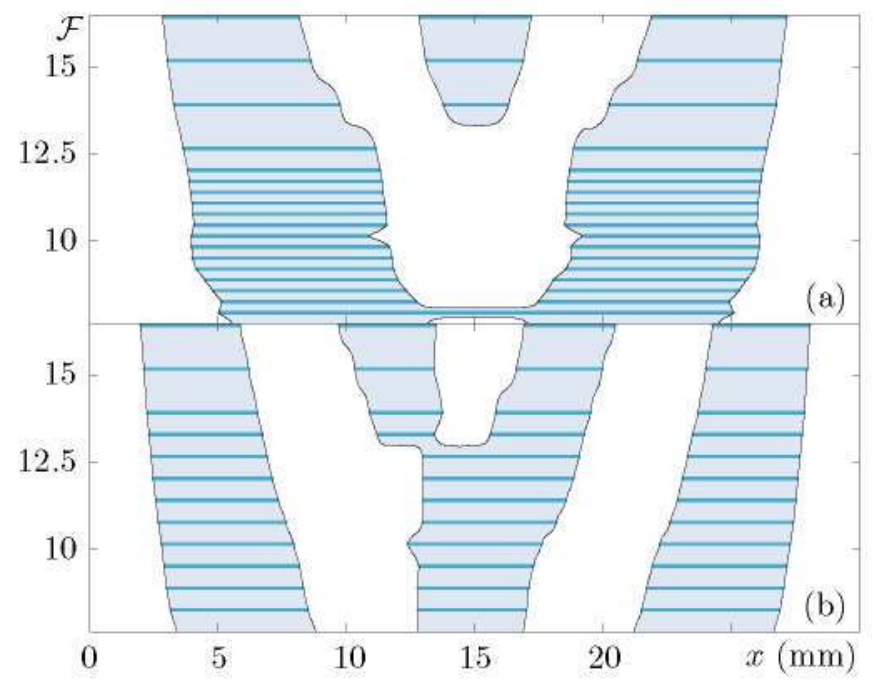

FIG. 10. Density distribution (silicone oil shaded; darker lines show resolution used) along the horizontal midline of the final steady state as a function of the vibrational parameter $\mathcal{F}$ for $L=30 \mathrm{~mm}$ and container heights (a) $2 H=3.75 \mathrm{~mm}$ and (b) $2 H=1.875 \mathrm{~mm}$. The thinner layer not only displays more asymmetry, but frustrates the development of patterns with large wavelengths: modes with $\langle\mathcal{C}\rangle=1,2$ are not observed when $2 H=1.875 \mathrm{~mm}$.

undergoes the same process, while the first column (on the right) stabilizes and the vigorous motion of the interface is transported inwards in preparation for another collision with the upper wall. In this manner, successive collisions of the interface with the upper and lower walls dynamically increase the wave number of the columnar pattern from $\langle\mathcal{K}\rangle \simeq 2$ at onset to $\langle\mathcal{K}\rangle=3$ in the final state, seen at $t=12.5 \mathrm{~s}$. Given this strongly nonlinear and asymmetric mechanism of column formation, the final structure is surprisingly symmetric, although not completely so.

Figure 10 shows the final steady fluid density distributions along the midline $y=0$ as function of the vibrational parameter in a container of length $L=30 \mathrm{~mm}$ for two different container heights: (a) $2 H=3.75 \mathrm{~mm}$ and (b) $1.875 \mathrm{~mm}$. In the thinner container, both of the first two modes, $\langle\mathcal{K}\rangle=1,2$, are prevented from developing into columnar structures by the process of repeated collisions with the upper and lower walls just described. The final columnar structure has evident asymmetry over most of the forcing range considered.

In the thicker container with $2 H=3.75 \mathrm{~mm}$, the $\langle\mathcal{K}\rangle=1$ mode is found only over a narrow range that depends on the complicated dynamics of the transient stage related with the movement of the contact line. The $\langle\mathcal{K}\rangle=2$ mode is considerably easier to obtain.

\section{B. Frozen wave instability in deep containers}

We now consider containers whose horizontal and vertical dimensions are comparable so $\Gamma \sim O(1)$. Compared to more shallow containers of the same length, the frozen wave columnar modes exist over a wider interval of vibrational parameters and greater asymmetry is observed in the final state, except very close to onset. The mechanisms behind this are described below.
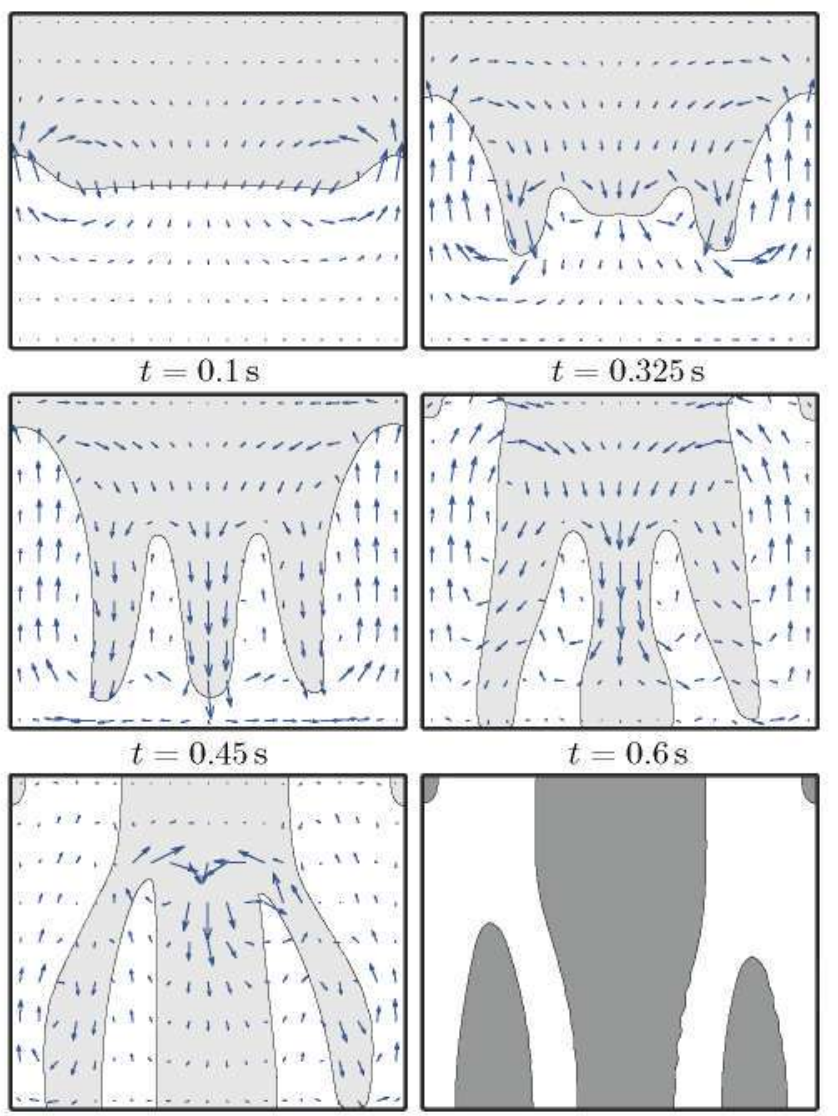

$t=0.8 \mathrm{~s}$

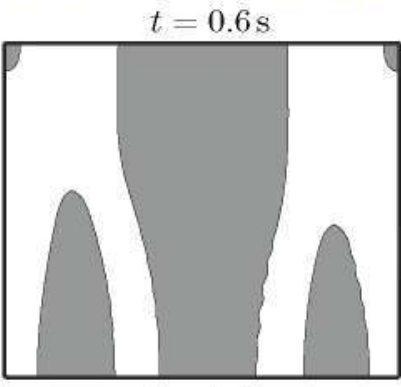

FIG. 11. Time evolution of the frozen wave instability in weightlessness in a $L \times 2 H=30 \mathrm{~mm} \times 30 \mathrm{~mm}$ rectangular container at $f=60 \mathrm{~Hz}, A=1.5 \mathrm{~mm}$, resulting in $\mathcal{F} \approx 37.97$. Snapshot times are labeled. The mean velocity field, averaged over one excitation period, is shown by scaled arrows (scaling not preserved between snapshots) for all but the final time. Asymmetric evolution driven by the harmonic motion of the lateral column crests is observed.

The snapshots shown in Fig. 11 illustrate the time evolution of a frozen wave pattern in a square container, $L \times 2 H=$ $30 \mathrm{~mm} \times 30 \mathrm{~mm}$, vibrated with $f=45 \mathrm{~Hz}$ and $A=1.5 \mathrm{~mm}$. When the forcing is first turned on, frozen waves grow in the same manner described in Sec. III A, with larger deformation near the lateral walls and smaller perturbations in the center of the container (see the snapshots for $t=0.1 \mathrm{~s}$ and $t=0.325 \mathrm{~s}$ ). Compared to thinner containers of identical length (see Fig. 2) the development of the columnar structure takes somewhat longer. The longer columns and increased transient time amplify the effect of the harmonic waves, which are out of phase on each side, as well as other sources of asymmetry produced by the forcing. The final columnar patterns reflect this enhanced asymmetry, as seen in the snapshot at $t=0.6 \mathrm{~s}$, which shows one column on the left that has already reached the lower wall while its counterpart on the right has not. Substantial asymmetry persists in the final steady columnar structure, as seen for $t=1.4 \mathrm{~s}$.

The final columnar state $(t=1.4 \mathrm{~s})$ also has an asymmetric distribution of subharmonic interfacial waves, most prominent on the right side of the central column. These Faraday waves 


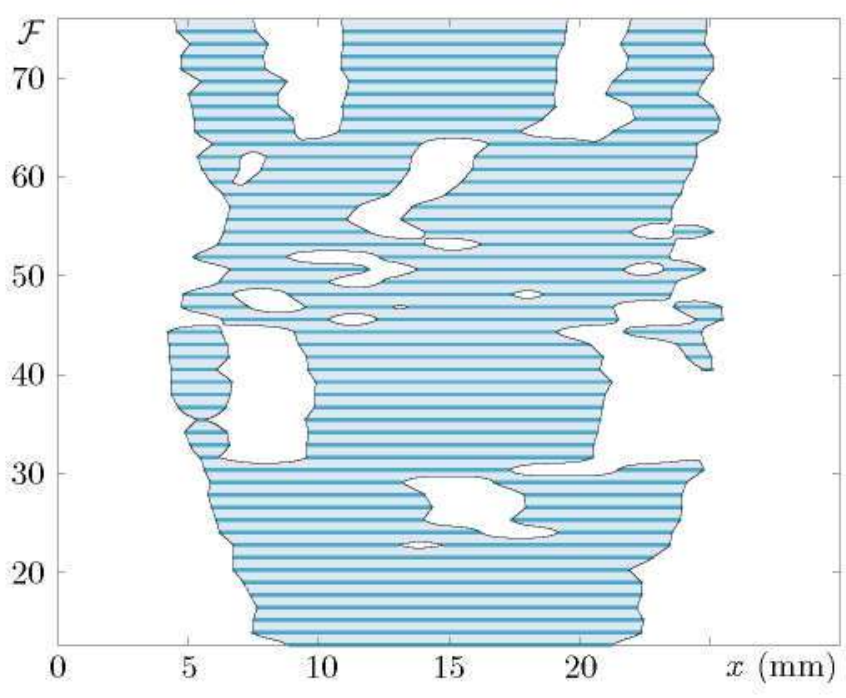

FIG, 12. Density distribution (silicone oil shaded; darker lines show resolution used) along the horizontal midline of the final steady state as a function of the vibrational parameter $\mathcal{F}$ for a deep container with $L \times 2 H=30 \times 30 \mathrm{~mm}$. Asymmetric behavior is observed quite generally for $\mathcal{F} \gtrsim 20$.

oscillate at half the forcing frequency (recall that the figures show an average over one excitation period) and introduce a second length scale to the frozen wave columnar structure. Solutions of this type were previously observed in microgravity experiments in horizontally vibrated containers with miscible liquids by Shevtsova et al. [26] and with immiscible liquids by Salgado Sánchez et al. [28].

Figure 12 shows the fluid (density) distribution as a function of the vibrational parameter $\mathcal{F}$. Other than a small range near onset for the $\langle\mathcal{K}\rangle=1$ mode, the increased asymmetry of the final structure in this deeper container is evident.

\section{DEPENDENCE OF THE INSTABILITY THRESHOLD ON ASPECT RATIO}

Here we focus on the onset of the first frozen wave mode with $\langle\mathcal{K}\rangle=1$ and compare the thresholds obtained numerically with the theoretical prediction for inviscid fluids in infinitely extended layers. Since these data are obtained by varying the length $L$, it is convenient to rescale using $H$ so that the nondimensional parameters characterizing the problem are independent of $L$. In analogy with Eqs. (9) and (11), we define the dimensionless velocity parameter and capillary number scaled with $H$ :

$$
\mathcal{F}_{H}=A \omega \sqrt{\frac{\left(\rho_{1}-\rho_{2}\right) H}{\sigma}}, \quad \mathrm{Ca}_{H}=v_{1} \sqrt{\frac{\left(\rho_{1}-\rho_{2}\right)}{\sigma H}} .
$$

The threshold prediction [20] for the inviscid system is

$$
(A \omega)^{2}=\frac{\left(\rho_{1}+\rho_{2}\right)^{3}}{2 \rho_{1} \rho_{2}\left(\rho_{1}-\rho_{2}\right)^{2}} \sigma k \tanh (k H),
$$

where $k$ is the dimensional wave number. Writing this in terms of $\mathcal{F}_{H}$ and using $k=2 \pi / L$ for the first mode gives the critical

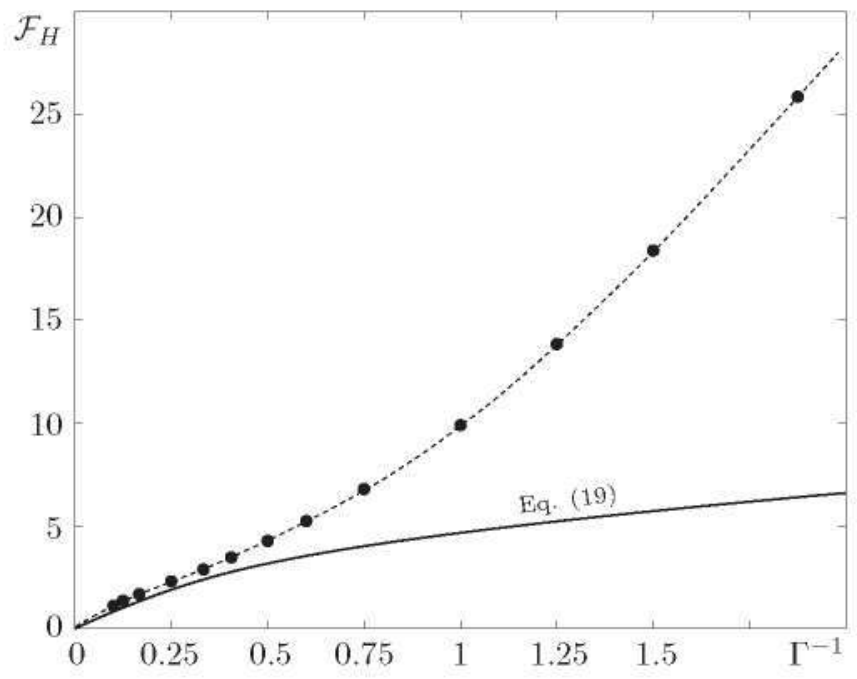

FIG. 13. Frozen wave threshold as a function of the inverse aspect ratio $\Gamma^{-1}$. Numerically obtained onset values (solid dots with dashed line for visual reference) were calculated for a capillary number $\mathrm{Ca}_{H}=0.0317$. The simulation values are slightly higher than the theoretical prediction (solid line) of Eq. (19) for $\Gamma^{-1} \lesssim 0.5$ but deviate substantially beyond that as finite size effects play a dominant role.

curve

$$
\mathcal{F}_{H}^{2}=\frac{\left(\rho_{1}+\rho_{2}\right)^{3}}{2 \rho_{1} \rho_{2}\left(\rho_{1}-\rho_{2}\right)}\left(\frac{\pi}{\Gamma}\right) \tanh \left(\frac{\pi}{\Gamma}\right),
$$

in terms of the aspect ratio $\Gamma$.

Figure 13 provides a comparison of the threshold predicted by Eq. (19) (shown with a solid line) and the results of Sec. IV (marked with solid dots). For $\Gamma^{-1} \lesssim 0.5$ the calculated onset values are about $10 \%-20 \%$ higher, but reasonably close to the theoretical prediction, with the difference likely explained by viscosity [17] and possibly also vibroequilibria effects [39-41]. As $\Gamma^{-1}$ becomes larger than 0.5 (short containers), the frozen wave instability is increasingly delayed by finite-size effects, diverging decisively from the theoretical prediction.

\section{CONCLUSIONS}

An extensive investigation of the columnar patterns triggered by the frozen wave instability in horizontally vibrated immiscible layers in weightless conditions was carried out numerically. The corresponding time-dependent problem was solved using a level-set formulation, as described in Sec. II. Our principal interest was in determining how pattern selection is affected by the finite size of the container, particularly for experimental configurations and liquids similar to those considered in Salgado Sánchez et al. [28].

The evolution of the interface after the frozen wave instability was described in Sec. III, including the column growth cycle defining mode transitions. An instability diagram showing the selected wave number for a given forcing was presented. In contrast to the continuous monotonic dependence of wave number on forcing predicted by linear inviscid theory neglecting lateral boundaries [20], the finite domain selects 
distinct solution branches that persist over finite intervals of the forcing.

The consequences of varying container length for fixed height were considered in Sec. IV. In particular, the frozen wave threshold decreases with increasing length, consistent with the requirement of inviscid theory $[13,20]$ that it vanish in the infinitely extended case. The onset of frozen waves in short containers is significantly delayed. In longer containers, where higher wave numbers can be studied, an interesting transition was found. The approximately symmetric (central) column growth cycle governing mode transitions at lower forcing is replaced at a certain point by a more complex, asymmetric column growth cycle, with the loss of average symmetry clearly visible in the mean velocity field.

The results of varying container height for fixed length were discussed in Sec. V. In thin containers, the development of large wavelength patterns is frustrated by repeated collisions with the upper and lower boundaries, where surface and contact forces play a key role. This highly nonlinear and asymmetric process acts to increase the average wave number of the final columnar structure, which may be relatively symmetric nonetheless. In thick containers, the asymmetry of the forcing and the associated harmonic surface waves, which are out of phase at the left and right endwalls, is amplified by the longer transient growth time, leading to final columnar structures with more asymmetry.

The onset values obtained from numerical simulations for the lowest $\mathcal{K}=1$ mode were quantitatively compared with the prediction for inviscid, extended layers of fluid [20] for different aspect ratios $\Gamma$. Reasonable agreement was found for $\Gamma \gtrsim 2$, with a small delay likely due to viscosity [17], but for $\Gamma \lesssim 2$, a more substantial increase in onset was seen, suggesting a limit for the applicability of theoretical results relying on the neglect of lateral boundaries.

Several aspects of the results described here suggest themselves for further investigation, an effort that will be under- taken separately. First, the vibroequilibria effect, which was mentioned briefly, clearly has a role in selecting the final interfacial structure. Evidence of this can be found in the fact that the denser liquid (FC-40) always ends up along the lateral walls, despite the fact that the frozen wave instability does not select for this. The vibroequilibria effect, however, does imply that the denser fluid should move up these walls rather than down them [39-41]. This effect also partly explains the difficulty of accurately measuring the frozen wave threshold in very long containers.

Finally, the manner in which the forcing is initiated is undoubtedly important. Here, motivated by parabolic flight experiments [28], the forcing was turned on relatively quickly. This led to larger disturbances and more rapid growth of the frozen waves near the lateral walls, with the central column being the last to develop and the most likely to suffer defects due to wave-number frustration, at least with forcing amplitudes below that of the asymmetric regime. In fact, the location of the column-generating defects in the more disordered asymmetric regime is, presumably, also related to the forcing function used and merits further investigation as well, particularly regarding the transition to the extended limit where discrete mode transitions are not anticipated.

\section{ACKNOWLEDGMENTS}

The work of Y.A.G. and V.S. was supported by the PRODEX programme of the Belgian Federal Science Policy Office. The work of P.S.S. and J.P. was supported by the Ministerio de Economía y Competitividad under Project No. ESP2015-70458-P. We also thank the Departamento de Aeronaves y Vehículos Espaciales in the Escuela Técnica Superior de Ingeniería Aeronáutica y del Espacio, Universidad Politécnica de Madrid for supporting the stay of P.S.S. at the Microgravity Research Centre, Université Libre de Bruxelles.
[1] M. Faraday, Philos. Trans. R. Soc. Lond. 121, 299 (1831).

[2] J. Miles and D. M. Henderson, Annu. Rev. Fluid Mech. 22, 143 (1990).

[3] K. Kumar and L. S. Tuckerman, J. Fluid Mech. 279, 49 (1994).

[4] H. Arbell and J. Fineberg, Phys. Rev. E 65, 036224 (2002).

[5] N. Prinet, D. Juric, and L. S. Tuckerman, J. Fluid Mech. 635, 1 (2009).

[6] G. H. Wolf, Phys. Rev. Lett. 24, 444 (1970).

[7] Y. A. Gaponenko, M. M. Torregrosa, V. Yasnou, A. Mialdun, and V. Shevtsova, J. Fluid Mech. 784, 342 (2015).

[8] C. J. R. Garrett, J. Fluid Mech. 41, 837 (1970).

[9] F. Varas and J. M. Vega, J. Fluid Mech. 579, 271 (2007).

[10] I. Tinao, J. Porter, A. Laveron-Simavilla, and J. Fernández, Phys. Fluids 26, 024111 (2014).

[11] J. M. Perez-Gracia, J. Porter, F. Varas, and J. M. Vega, J. Fluid Mech. 739, 196 (2014).

[12] P. Salgado Sánchez, J. Porter, I. Tinao, and A. LaverónSimavilla, Phys. Rev. E 94, 022216 (2016).

[13] D. V. Lyubimov and A. A. Cherepanov, Fluid Dyn. 21, 849 (1986).
[14] M. V. Khenner, D. V. Lyubimov, T. S. Belozerova, and B. Roux, Eur. J. Mech. B 18, 1085 (1999).

[15] R. Wunenburger, P. Evesque, C. Chabot, Y. Garrabos, S. Fauve, and D. Beysens, Phys. Rev. E 59, 5440 (1999).

[16] D. V. Lyubimov, M. Khenner, and M. M. Shotz, Fluid Dyn. 33, 318 (1998).

[17] E. Talib, S. V. Jalikop, and A. Juel, J. Fluid Mech. 584, 45 (2007).

[18] S. V. Jalikop and A. Juel, J. Fluid Mech. 640, 131 (2009).

[19] H. N. Yoshikawa and J. E. Wesfreid, J. Fluid Mech. 675, 249 (2011).

[20] T. P. Lyubimova, A. O. Ivantsov, Y. Garrabos, C. Lecoutre, G. Gandikota, and D. Beysens, Phys. Rev. E 95, 013105 (2017).

[21] D. Beysens, Y. Garrabos, D. Chatain, and P. Evesque, Europhys, Lett. 86, 16003 (2009).

[22] G. Gandikota, D. Chatain, S. Amiroudine, T. Lyubimova, and D. Beysens, Phys. Rev. E 89, 013022 (2014).

[23] G. Gandikota, D. Chatain, S. Amiroudine, T. Lyubimova, and D. Beysens, Phys. Rev. E 89, 012309 (2014). 
[24] D. Sharma, A. Erriguible, G. Gandikota, D. Beysens, and S. Amiroudine, Phys. Rev. Fluids 4, 033401 (2019).

[25] Y. A. Gaponenko, M. M. Torregrosa, V. Yasnou, A. Mialdun, and V. Shevtsova, Soft Matter 11, 8221 (2015).

[26] V. Shevtsova, Y. A. Gaponenko, V. Yasnou, A. Mialdun, and A. Nepomnyashchy, J. Fluid Mech. 795, 409 (2016).

[27] Y. A. Gaponenko, A. Mialdun, and V. Shevtsova, Phys. Fluids 30, 62103 (2018).

[28] P. Salgado Sánchez, V. Yasnou, Y. Gaponenko, A. Mialdun, J. Porter, and V. Shevtsova, J. Fluid Mech. 865, 850 (2019).

[29] E. Olsson and G. Kreiss, J. Comput. Phys. 210, 225 (2005).

[30] J. A. Sethian, Level Set Methods and Fast Marching Methods: Evolving Interfaces in Computational Geometry, Fluid Mechanics, Computer Vision, and Materials Science, Cambridge monographs on applied and computational mathematics (Cambridge University Press, Cambridge, 1999).

[31] D. B. Kothe, R. C. Mjolsness, and M. D. Torrey, RIPPLE: A Computer Program for Incompressible Flows with Free Sur- faces, Report LA-12007-MS (Los Alamos National Laboratory, Los Alamos, NM, 1991).

[32] S. Someya and T. Munakata, J. Cryst. Growth 275, 343 (2005).

[33] S. Erlicher, L. Bonaventura, and O. Bursi, Comput. Mech. 28, 83 (2002).

[34] I. Harari and T. J. Hughes, Comput. Methods Appl. Mech. Eng. 97, 157 (1992).

[35] R. Codina, Comput. Methods Appl. Mech. Eng. 110, 325 (1993).

[36] A. V. Burnysheva, D. V. Lyubimov, and T. P. Lyubimova, Fluid Dyn. 46, 1000 (2011).

[37] B. D. Dore, Math. Proc. Camb. Philos. Soc. 74, 333 (1973).

[38] V. Shevtsova, Y. A. Gaponenko, V. Yasnou, A. Mialdun, and A. Nepomnyashchy, Langmuir 31, 5550 (2015).

[39] R. F. Ganiev, V. D. Lakiza, and A. S. Tsapenko, Sov. Appl. Mech. 13, 499 (1977).

[40] J. Fernández, I. Tinao, J. Porter, and A. Laveron-Simavilla, Phys. Fluids 29, 24108 (2017).

[41] J. Fernández, P. Salgado Sanchez, I. Tinao, J. Porter, and J. M. Ezquerro, Microgr. Sci. Technol. 29, 351 (2017). 